4 Raghu G, Collard HR, Egan JJ, et al. An official ATS/ERS/JRS/ALAT statement: idiopathic pulmonary fibrosis: evidence-based guidelines for diagnosis and management. Am J Respir Crit Care Med 2011; 183: 788-824.

5 Lee JS, Song JW, Wolters PJ, et al. Bronchoalveolar lavage pepsin in acute exacerbation of idiopathic pulmonary fibrosis. Eur Respir J 2012; 39: 352-358.

6 Allen JN. Drug-induced eosinophilic lung disease. Clin Chest Med 2004; 25: 77-88.

7 Solomon J, Schwarz M. Drug-, toxin-, and radiation therapy-induced eosinophilic pneumonia. Semin Respir Crit Care Med 2006; 27: 192-197.

8 Yoshioka S, Mukae H, Ishii H, et al. [A case of drug-induced pneumonia possibly associated with simvastatin]. Nihon Kokyuki Gakkai Zasshi 2005; 43: 600-604.

9 European Medicines Agency. Esbriet: EPAR Product Information. www.ema.europa.eu/docs/en_GB/document_ library/EPAR_-_Product_Information/human/002154/WC500103049.pdf Date last updated: 2015. Date last accessed: September 11, 2015.

10 Campainha S, Nogueira C, Costa F, et al. Not yet known side effects of pirfenidone in the treatment of idiopathic pulmonary fibrosis? Rev Port Pneumol 2016; 22: 126-127.

\title{
Can intermediate monocytes predict response to infliximab therapy in sarcoidosis?
}

\author{
To the Editor:
}

Sarcoidosis is a systemic granulomatous disease of unknown origin that can cause a variety of symptoms, but most often affects the lungs [1]. Because sarcoidosis is self-limiting in the majority of patients, not all patients require therapy [2]. In case of severe sarcoidosis, first-line therapy consists of prednisone, with methotrexate or azathioprine being the most commonly used second-line options [3]. Infliximab, a monoclonal anti-tumour necrosis factor (TNF) drug, is an effective third-line therapeutic for severe sarcoidosis $[4,5]$.

The exact nature and order of immunological events leading to the formation of sarcoid granulomas remains unknown. Granulomas consist of highly differentiated mononuclear phagocytes (mainly activated macrophages) and lymphocytes [1]. Macrophages of sarcoidosis patients are known to produce excessive amounts of TNF compared with healthy controls $[6,7]$. The importance of TNF is underlined by the success of infliximab therapy. As monocytes are the precursors of tissue macrophages, monocytes might be a key player in this process. A promising new therapeutic strategy for sarcoidosis is the combined monocyte and granulocyte apheresis [8]. Although only seven patients were included in a pilot study, the positive effects underline the importance of monocytes in the sarcoidosis disease process.

CD16-expressing monocytes are expanded in various immune-mediated diseases, such as rheumatoid arthritis, diabetes, atherosclerosis, bacterial infections and HIV [9]. High percentages of CD16-expressing monocytes were also found in sarcoidosis patients when compared with controls $[10,11]$. CD16-expressing monocytes, and intermediate monocytes in particular, fulfil a pro-inflammatory role by producing TNF as well as other cytokines [12]. We have previously shown that sarcoidosis patients have a significantly higher percentage of the pro-inflammatory intermediate monocytes than healthy controls [13], an observation that has been confirmed recently by Hofer et al. [14]. To date, the few available studies investigating monocytes in sarcoidosis have focused solely on monocyte subsets while any potential relationship between disease activity or response to therapy has not yet been addressed.

In our present study, we analysed whether monocyte subsets in blood can predict the clinical response of sarcoidosis patients to therapy with infliximab (Remicade; Centocor Inc., Malvem, PA, USA). To this end we have studied a selected group of patients with severe sarcoidosis, who were unresponsive to first- and second-line treatment or experienced severe side-effects. These patients received infliximab intravenously according to a standardised protocol starting with $5 \mathrm{mg} \cdot \mathrm{kg}^{-1}$ at weeks 0 and 2 and then every 4 weeks for a period of 26 weeks. This study is part of a prospective, open-label cohort study by VorselaARs et al. [15] evaluating the efficacy of infliximab in sarcoidosis. The institutional review board and the ethics committee approved the study and all patients gave written informed consent.

Peripheral mononuclear blood cells from 38 sarcoidosis patients and 18 healthy controls were stained for CD14, CD16 (eBioscience, San Diego, CA, USA) and HLA-DR (human leukocyte antigen-antigen D related) 
(BD Biosciences, San Diego, CA, USA) before the start of therapy (baseline) and at 14 and 26 weeks of treatment using flow cytometry. Data were acquired on a FACSCalibur (BD Biosciences) and analysed using FlowJo software (Tree Star, Ashland, OR, USA). We used a gating strategy of FSC/SSC, HLA-DR/SSC and then CD14/CD16 (figure 1a). Monocyte cell counts were determined the day before infusion.

Response to infliximab was defined as a reduction in biomarker levels (soluble interleukin-2 receptor or angiotensin converting enzyme) or maximum standardised uptake value on ${ }^{18}$ F-fluorodeoxyglucose positron emission tomography $\geqslant 40 \%$ at week 26 compared with baseline [15]. Using these criteria, 30 patients were classified as responders and eight as non-responders. Flow cytometry data from two responders and one non-responder were missing either at week 14 or 26.

In order to address the question whether monocytes can predict the response to infliximab therapy, we first investigated whether the absolute numbers and the percentages of monocytes and its subsets differed at baseline (figure $1 \mathrm{~b}$ and $\mathrm{c}$ ). The absolute number of total monocytes at baseline was increased in responders $\left(0.64 \times 10^{9}\right.$ cells $\left.\cdot \mathrm{L}^{-1}\right)$ compared with non-responders $\left(0.46 \times 10^{9}\right.$ cells $\left.\cdot \mathrm{L}^{-1}, \mathrm{p}=0.019\right)$ (figure $\left.1 \mathrm{~b}\right)$. The absolute number of intermediate monocytes at baseline was also increased in responders $\left(0.023 \times 10^{9}\right.$ cells $\left.\cdot \mathrm{L}^{-1}\right)$ compared with non-responders $\left(0.011 \times 10^{9}\right.$ cells $\left.\cdot \mathrm{L}^{-1}, \mathrm{p}=0.011\right)$. Interestingly, the percentage of intermediate monocytes at baseline was significantly increased in responders $(3.43 \%)$ compared with controls $(1.82 \%$, $\mathrm{p}<0.001$ ) (figure 1c). When comparing this group of severe sarcoidosis patients with healthy controls at baseline we found a significant increase in the percentage of intermediate monocytes $(3.24 \%$ versus $1.82 \%$, $\mathrm{p}<0.001$ ) (figure 1e), which confirms our data and earlier data $[13,14]$. In contrast to what was found by Hofer et al. [14], we found no differences in percentages of intermediate monocytes between males $(n=24)$ and females $(\mathrm{n}=14, \mathrm{p}=0.71)$.

Furthermore, we studied whether the absolute numbers and percentages of monocytes and its subsets changed during infliximab therapy. Figure 1d shows an example of how the patterns of monocyte subsets of a responder changed during therapy.

Non-responders showed a significant increase in absolute numbers of intermediate monocytes after 26 weeks of therapy (from $0.009 \times 10^{9}$ cells $\cdot \mathrm{L}^{-1}$ to $0.027 \times 10^{9}$ cells $\cdot \mathrm{L}^{-1} ; \mathrm{p}=0.028$ ). Moreover, non-responders showed significant increases during 26 weeks of therapy in both absolute numbers of total monocytes $(\mathrm{p}=0.027)$ and absolute numbers of classical monocytes $(\mathrm{p}=0.028)$. Responders showed a significant decrease in non-classical monocytes after 14 and 26 weeks of therapy (from $0.100 \times 10^{9}$ cells $\cdot \mathrm{L}^{-1}$ to $0.074 \times 10^{9}$ cells $\cdot \mathrm{L}^{-1}$ to $0.079 \times 10^{9}$ cells $\cdot \mathrm{L}^{-1}, \mathrm{p}=0.001$ and $\mathrm{p}=0.011$, respectively).

Sarcoidosis patients as a total group showed an increase in the percentage of intermediate monocytes from the start of therapy to 14 weeks of therapy (from $3.2 \%$ to $4.1 \% ; \mathrm{p}=0.010$ ), but fell back to baseline at 26 weeks (figure 1e). Responders also showed an initial slight increase in the percentage of intermediate monocytes after 14 weeks of therapy, but fell back to baseline at 26 weeks. Non-responders showed a significant increase in intermediate monocytes after 26 weeks of therapy (from $2.23 \%$ to $3.99 \% ; p=0.018$ ) (figure 1f). Additionally, sarcoidosis patients as a total group showed a significant decrease in the percentage of non-classical monocytes after 14 and 26 weeks of therapy (from $15.4 \%$ to $12.6 \%$ to $12.3 \%$; $\mathrm{p}=0.003$ and $\mathrm{p}=0.006$, respectively) (figure $1 \mathrm{~g}$ ). Responders also showed a significant decrease in non-classical monocytes after 14 weeks and 26 weeks of therapy (from $15.6 \%$ to $13.5 \%$ to $12.7 \%$; $=0.012$ and $\mathrm{p}=0.036$, respectively) (figure $1 \mathrm{~h}$ ). Non-responders showed a decrease in the percentage of non-classical monocytes as well, but this was not significant.

In conclusion, we show that responders to infliximab therapy have more intermediate monocytes at baseline compared with non-responders. The high level of intermediate monocytes possibly reflects a high inflammatory state, the latter is correlated with a better clinical response to infliximab [15]. The intermediate monocytes of non-responders increase significantly during the initial phase of therapy with infliximab. A similar initial increase of intermediate monocytes following infliximab therapy was seen in patients with Crohn's disease after three infusions [12]. The authors of that work proposed that it could be a compensatory response of the host to TNF depletion, as intermediate monocytes are high TNF producers [12]. Long-term effects were not studied by NAZARETH et al. [12].

At baseline, total monocyte numbers (percentage as well as absolute) are decreased in non-responders compared with responders, while during therapy non-responders showed a significant increase of total monocytes. During therapy the non-classical, also pro-inflammatory, monocytes normalise to levels of healthy controls.

Our findings show that the intermediate monocyte population can be used as an indicator for responsiveness to anti-TNF therapy in sarcoidosis. Further investigation of the ratio of monocyte subsets and their function may help us to understand the disease process and predict response to therapy. 


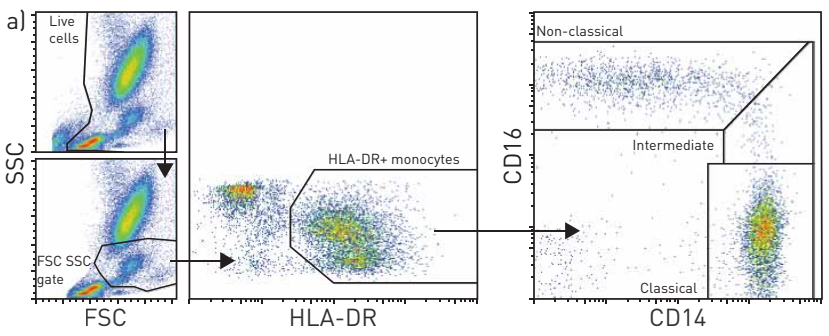

d)

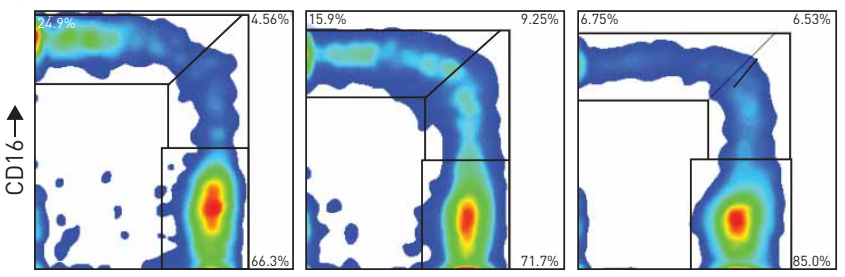

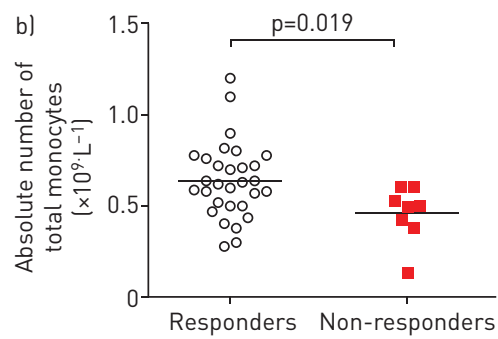

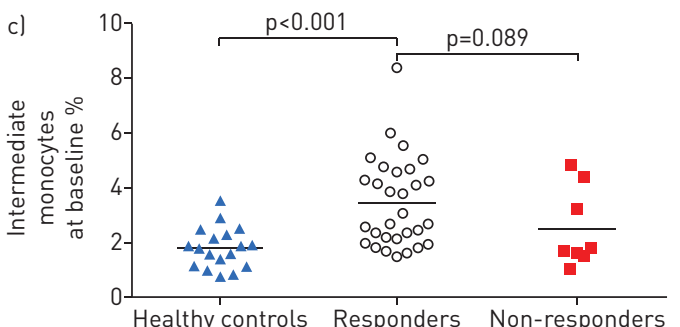

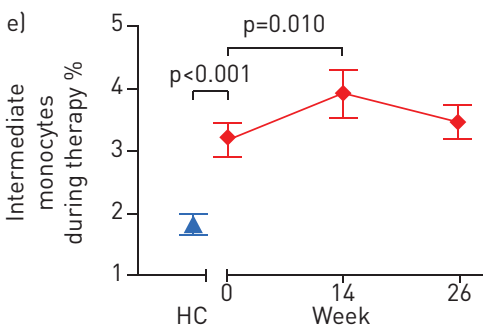

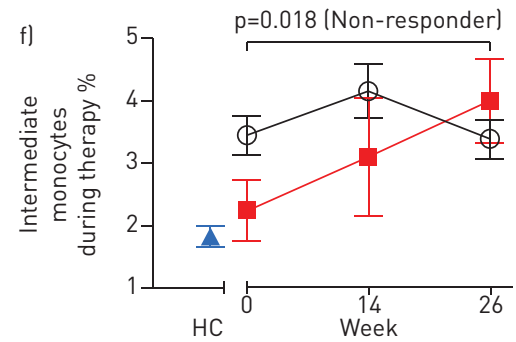

- Healthy controls

$\dashv$ Sarcoidosis patients

๑ Responders

- Non-responders
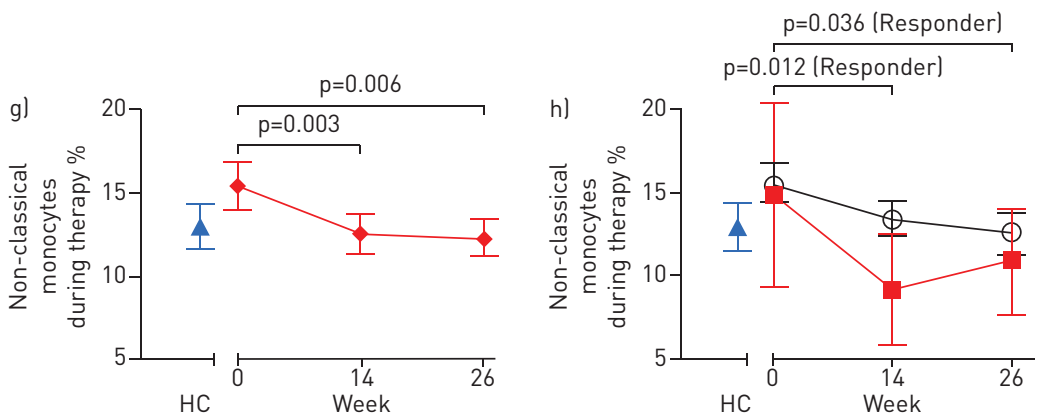

FIGURE 1 Changes in monocytes and its subsets in human peripheral blood cells at the start and during therapy with infliximab. Blood samples of 18 healthy controls and 38 sarcoidosis patients (30 responders and eight non-responders) were collected before the start of therapy at week 0 (baseline) and during therapy at week 14 and 26 prior to infusion with infliximab. Data from two responders and one non-responder were missing either at week 14 or 26. a) Peripheral blood cells were stained for HLA-DR (human leukocyte antigen-antigen D related) CD14 and CD16. Monocytes were first gated based on FSC $\times S S C$, then on HLA-DR $\times S S C$ and finally subdivided into three subsets based on CD14/CD16 expression. Percentages of HLA-DR-positive monocytes were calculated as percentage of leukocytes, and percentages of monocyte subsets as percentages of HLA-DR-positive monocytes. FSC: forward scatter; SSC: side scatter. b) The absolute number of total monocytes was decreased in non-responders to therapy compared with responders $(p=0.019)$. $c)$ The percentage of intermediate monocytes was increased in responders to therapy compared with healthy controls $(p<0.001)$. d) An example of how the patterns of monocyte subsets changed during infliximab therapy in a responder. e) Intermediate monocytes were increased in sarcoidosis patients compared with healthy controls (HC) ( $p<0.001)$. The patients showed an increase in the percentage of intermediate monocytes after 14 weeks of therapy $(p=0.010)$, but fell back to baseline at 26 weeks. f) Non-responders showed a significant increase in the percentage of intermediate monocytes during therapy at week 26 ( $p=0.018$ ). g) Sarcoidosis patients showed a decrease in the percentage of non-classical monocytes at week 14 and 26 of therapy ( $p=0.003$ and $p=0.006$, respectively). h) Responders showed a significant decrease in the percentage of non-classical monocytes during therapy at week 14 and 26 ( $p=0.012$ and $p=0.036$, respectively). Differences in baseline expression levels between groups were compared using the Mann-Whitney U-test. Differences between expression patterns at the start of therapy (week 0 ) and at 14 and 26 weeks of therapy were compared using the Wilcoxon signed-rank test. All were considered statistically significant at $p$-values $<0.05$.

@ERSpublications

Responders to infliximab therapy have more intermediate monocytes at baseline compared with non-responders http://ow.ly/fMLp300oVrO

Daniëlle Hijdra $^{1,2,6}$, Adriane D.M. Vorselaars ${ }^{1,6}$, Heleen A. Crommelin ${ }^{1,3}$, Coline H.M. van Moorsel ${ }^{1,4}$, Bob Meek ${ }^{2}$, Anke M.E. Claessen ${ }^{2}$, Ger T. Rijkers ${ }^{2,5}$ and Jan C. Grutters ${ }^{1,4}$ 
${ }^{1}$ Interstitial Lung Diseases Centre of Excellence, Dept of Pulmonology, St. Antonius Hospital, Nieuwegein, The Netherlands. ${ }^{2}$ Dept of Medical Microbiology and Immunology, St. Antonius Hospital, Nieuwegein, The Netherlands. ${ }^{3}$ Dept of Clinical Pharmacy, St. Antonius Hospital, Nieuwegein, The Netherlands. ${ }^{4}$ Division of Heart \& Lungs, University Medical Centre Utrecht, Utrecht, The Netherlands. ${ }^{5}$ Dept of Sciences, Roosevelt Academy, Middelburg, The Netherlands. ${ }^{6}$ Both authors contributed equally.

Correspondence: Adriane D.M. Vorselaars, Interstitial Lung Diseases Centre of Excellence, Dept of Pulmonology, St. Antonius Hospital, Koekoekslaan 1, 3435 CM Nieuwegein, The Netherlands. E-mail: a.vorselaars@antoniusziekenhuis.nl

Received: April 072016 | Accepted after revision: May 02 2016 | First published online: July 072016

Clinical trials: This study is registered at www.trialregister.nl with identifier number NTR3895.

Conflict of interest: None declared.

Support statement: This work was supported by the Innovation Fund of the St. Antonius Hospital (Nieuwegein, The Netherlands).

\section{References}

1 Statement on sarcoidosis. Joint Statement of the American Thoracic Society (ATS), the European Respiratory Society (ERS) and the World Association of Sarcoidosis and Other Granulomatous Disorders (WASOG) adopted by the ATS Board of Directors and by the ERS Executive Committee, February 1999. Am J Respir Crit Care Med 1999; 160: 736-755.

2 Valeyre D, Prasse A, Nunes H, et al. Sarcoidosis. Lancet 2014; 383: 1155-1167.

3 Vorselaars AD, Cremers JP, Grutters JC, et al. Cytotoxic agents in sarcoidosis: which one should we choose? Curr Opin Pulm Med 2014; 20: 479-487.

4 Baughman RP, Drent M, Kavuru M, et al. Infliximab therapy in patients with chronic sarcoidosis and pulmonary involvement. Am J Respir Crit Care Med 2006; 174: 795-802.

5 Crommelin HA, Vorselaars AD, van Moorsel CH, et al. Anti-TNF therapeutics for the treatment of sarcoidosis. Immunotherapy 2014; 6: 1127-1143.

6 Ziegenhagen MW, Rothe ME, Zissel G, et al. Exaggerated TNF- $\alpha$ release of alveolar macrophages in corticosteroid resistant sarcoidosis. Sarcoidosis Vasc Diffuse Lung Dis 2002; 19: 185-190.

7 Zheng L, Teschler H, Guzman J, et al. Alveolar macrophage TNF- $\alpha$ release and BAL cell phenotypes in sarcoidosis. Am J Respir Crit Care Med 1995; 152: 1061-1066.

8 Olsen HH, Muratov V, Cederlund K, et al. Therapeutic granulocyte and monocyte apheresis (GMA) for treatment refractory sarcoidosis: a pilot study of clinical effects and possible mechanisms of action. Clin Exp Immunol 2014; 177: $712-719$.

9 Ziegler-Heitbrock L. The CD14+ CD16+ blood monocytes: their role in infection and inflammation. J Leukoc Biol 2007; 81: 584-592.

10 Heron M, Grutters JC, van Velzen-Blad H, et al. Increased expression of CD16, CD69, and very late antigen-1 on blood monocytes in active sarcoidosis. Chest 2008; 134: 1001-1008.

11 Okamoto H, Mizuno K, Horio T. Circulating CD14+ CD16+ monocytes are expanded in sarcoidosis patients. J Dermatol 2003; 30: 503-509.

12 Nazareth N, Magro F, Silva J, et al. Infliximab therapy increases the frequency of circulating CD16(+) monocytes and modifies macrophage cytokine response to bacterial infection. Clin Exp Immunol 2014; 177: 703-711.

13 Hijdra D, Vorselaars AD, Grutters JC, et al. Differential expression of TNFR1 (CD120a) and TNFR2 (CD120b) on subpopulations of human monocytes. J Inflamm (Lond) 2012; 9: 38-9255-9-38.

14 Hofer TP, Zawada AM, Frankenberger M, et al. Characterization of subsets of the CD16-positive monocytes: impact of granulomatous inflammation and M-CSF-receptor mutation. Blood 2015; 126: 2601-2610.

15 Vorselaars AD, Crommelin HA, Deneer VH, et al. Effectiveness of infliximab in refractory FDG PET-positive sarcoidosis. Eur Respir J 2015; 46: 175-185. 